\title{
Cloning and Overexpression of GmDREB2 Gene from a Vietnamese Drought-resistant Soybean Variety
}

\author{
Dao Xuan Tan ${ }^{1}$, Ho Manh Tuong ${ }^{2}$, Vu Thi Thu Thuy ${ }^{1}$, Le Van Son ${ }^{2}$ and Chu Hoang Mau ${ }^{*}$ \\ ${ }^{1}$ Thai Nguyen University of Education; Vietnam. ${ }^{2}$ Institute of Biotechnology, Vietnam Academy of Science and \\ Technology; Vietnam
}

\begin{abstract}
This work studied the amplification, cloning and determination of the GmDREB2 gene from the soybean cultivar DT2008 and five Vietnamese local soybean cultivars (DT26, DT51, DVN5,CB, CBD) and designed the vector carrying the structure containing GmDREB2 gene from cultivar DT2008 (best drought tolerant). The coding region of GmDREB2 gene isolated from six soybean cultivars was 480 nucleotides in length, encoding 159 amino acids. The recombinant structure was designed as 35S-GmDREB2-c-myc and its expression was analysed in transgenic tobacco plants. Recombinant DREB2 protein was expressed in five transgenic tobacco lines with molecular weights close to $20 \mathrm{kDa}$. During the drought conditions, the proline accumulation of the transgenic tobacco lines was higher than on wild-type (WT) plants, with the rate from 211.17 to $332.44 \%$ after five days of drought stress, and from 262.79 to $466.04 \%$ after nine days of drought stress. The two lines, TG2 and TG12 had the highest increase rate. These results provided the basis to generate drought-tolerant soybean plants by GmDREB2 overexpression.
\end{abstract}

Key words: drought-resistant, GmDREB2 gene, gene transfer, soybean, transcription factors

\section{INTRODUCTION}

Soybean (Glycine max (L.) Merrill) is a species of legume, which has economic values and high nutrition, plays a key role in improving the soil fertility and the sustainable usage of arable land resources. It is globally an important crop, including in Vietnam. However, soybean is considered one of the most drought sensitive crops, hence, studies for enhancing the drought tolerant ability are significant in improving the productivity and qualities of soybean. Some genes have been described as having responded to drought stress in the transcription level ( $\mathrm{Li}$ et al. 2005). In 1994, Yamaguchi and Shinozaki discovered a cis- acting element, which responded to dehydration and was controlled by the
COR78/RD29A promoter involved in the drought, low temperature and high salt stresses.

Trans factor- DREB protein can bind to cis- acting element to stimulate the expression in the plants by stress signal from the environment. DREB protein belongs to the transcript factor AP2/ERF family, which can control the expression of genes induced by the drought, high salt and low temperature stresses, resulting in the tolerant ability of environment stresses in the plants. DREB genes have been isolated from different crops such as Arabidopsis, Oryza sativa (L.), Zea mays (L.), Triticum aestivum and other crops (Li et al. 2005; Chen et al. 2007; Andeani et al. 2009). At least 10 members of GmDREB subfamily were identified in the genome of soybean (GmDREBa, GmDREBb, GmDREBc, GmDREB1, GmDREB2, GmDREB3, GmDREB5, GmDREB6, and

*Author for correspondence: chuhoangmau@tnu.edu.vn 
GmDREB7) (Riechmann et al. 2000). Chen et al. (2007) isolated GmDREB2 gene from soybean and based on the sequence identity of the AP2 domain, GmDREB2 gene was classified into A-5 subgroup in DREB gene subfamily. Upregulation of GmDREB2 gene is induced by the drought, high salt, and ABA environment. The expression of GmDREB2 gene in transgenic tobacco plants accumulated high levels of proline (Kobayashi et al. 2008). The nucleotide sequence of GmDREB2 gene was published in GeneBank by Wang et al. (2006). GmDREB2 was found in soybean under drought stress, low temperature and high salt conditions. These results indicated that GmDREB2 gene could improve the drought-tolerance of soybean (Chen et al. 2007; Kobayashi et al. 2008; Mizoi et al. 2013). This work aimed to study the cloning and expression of GmDREB2 gene from Vietnamese drought-resistant soybean cultivars in order to provide basis for improving the drought tolerance of soybean in Vietnam.

\section{MATERIAL AND METHODS}

\section{Material}

The Vietnamese soybean cultivars, labeled CB, CBD, DT26, DT51, DVN5 and DT2008 offered by the Vietnam Institute of Agricultural Genetics were used for isolation and molecular cloning of GmDREB2 gene. Cloning vector pBT, vector pRTRA7/3 containing the $35 \mathrm{~S}$ promoter and cmyc tag, pBI121 vector were provided by the Laboratory of Applied DNA Technology. A. tumefaciens CV58, N. tabacum K326 were provided by the Laboratory of Plant Cell Biotechnology, Institute of Biotechnology, Vietnam.

\section{Methods}

Total RNA of soybean was extracted using the Gene JET Plant RNA Purification Mini Kit (Thermo Scientific) according to the instructions of manufacturer. The cDNA was synthesized from the total RNA using RevertAid First Strand cDNA Synthesis Kit (Thermo Scientific). DREB2 cDNA was amplified by the PCR using primer pairs, which were designed from the nucleotide sequence in the GenBank database of DREB2 code number DQ054363 (Wang et al. 2006).

GmDREB2-NcoI:

5' CATGCCATGGATGGAAGAAGCGGGTTTAG 3' GmDREB2-NotI :

5' TTGCGGCCGCATCTTCAGGTTTGGGA 3'
The PCR reaction was done as following: $94^{\circ} \mathrm{C}$ for $4 \mathrm{~min}$, followed by 30 cycles of $94^{\circ} \mathrm{C}$ for $30 \mathrm{~s}$, $58^{\circ} \mathrm{C}$ for $30 \mathrm{~s}, 72^{\circ} \mathrm{C}$ for $30 \mathrm{~s}$, and 1 cycle of $72^{\circ} \mathrm{C}$ for $10 \mathrm{~min}$. The amplified PCR products were analyzed by electrophoresis on $1 \%$ agarose gel in 1X TAE electrophoresis buffer. The amplified products were cloned according to Sambrook and Russell (2001). PCR products were purified from agarose gel using DNA extraction kit (Fermentas) and ligated into $\mathrm{pBT}$, then were transformed into E.coli DH5 $\alpha$. Plasmid isolation was performed using the Plasmid Miniprep kit (Qiagen). The nucleotide sequence was determined using the sequencing machine ABI PRISM@ 3100 Advant Genetic Analyzer (Applied Biosystem). The analysis of nucleotide sequence was done by the BioEdit software and DNAstar.

\section{Construction of 35S- GmDREB2- c- myc}

The target gene from the cloning vector pBTGmDREB2 was cut out from the cloning vector pBT using restriction enzymes $\mathrm{NcoI}$ and NotI, then ligated into pRTRA 7/3, which carried 35S promoter and c-myc tag.

\section{Construction of transgenic vector and transferred into tobacco plants}

The recombinant structure 35S-GmDREB2-c-myc were cut out from the vector pRTRA, then ligated into pBI121 and transformed into E. coli DH5 $\alpha$. The recombinant vector was isolated following Sambrook and Russell method (2001), then was checked using the restriction enzyme HindIII. The existence of GmDREB2 gene was identified by the PCR using primer pairs GmDREB2NcoI/GmDREB2-NotI. The selected recombinant vector $\quad p B I 121 / 35 S-G m D R E B 2-c-m y c$ was transformed into A. tumefaciens CV58.

Transformation of $p$ BI121/35S-GmDREB2-c-myc into tobacco cultivars K326 by A.tumefaciens was performed following the protocol of Topping et al. (1998). The transgenic samples were regenerated on MS medium supplemented with BAP, cefotaxime $(500 \mathrm{mg} / \mathrm{L})$ and kanamycine $(50 \mathrm{mg} / \mathrm{L})$. To investigate the existence of the target gene, tobacco leaves were used for DNA extraction and PCR reaction with specific primer pairs, GmDREB2- NcoI/NotI. Transgenic tobacco plants, which were confirmed to be positive by PCR, were subjected for the analysis of expression of the recombinant GmDREB2 by Western Blot. 
Analysis of recombinant DREB2 protein in transgenic tobacco by Western blot

To extract total protein, $0.5 \mathrm{~g}$ leaves were crushed in liquid nitrogen and dissolved in $1.0 \mathrm{~mL}$ of PBS with $0.05 \%$ Tween 20 (PBS-T), then centrifuged at $13000 \mathrm{~g}$ for $15 \mathrm{~min}$. Proteins were denatured and run on $10 \%$ SDS-PAGE, then transferred to nitrocellulose membranes using Pierce G2 Fast Blotter $(25 \mathrm{~V}, 1.3 \mathrm{~mA}$ for $20 \mathrm{~min})$. Membranes were then blocked in the blocking solution $(5 \%$ skimmed milk in PBS-T) overnight, then incubated with primary antibody (c-myc) for $3 \mathrm{~h}$ by shaking at room temperature, followed by three times washing with PBS, then incubated with secondary antibody for $2 \mathrm{~h}$. Mouse monoclonal antibody to c-myc (Santa Cruz Biotech) was diluted in 5\% milk in PBS-T at 1:700. For secondary antibody, anti-mouse $\operatorname{IgG}$ antibody attached HRP (Horse Radish Peroxidase) was diluted in 5\% milk in PBS-T at 1:4000. Results were displayed using TMB (3,3',5,5'-tetramethyl benzidine) or DAB (3,3'-diaminobenzidine tetrahydrochloride).

Evaluation of drought tolerance of tobacco plants via analysis of concentrations of proline When tobacco plants had 5-6 leaves, artificial drought stress was nititiated. Proline levels in the leaves of transgenic lines and WT were analyzed after five and nine days of drought stress. Proline concentrations were determined by the method of Bates et al. (1973).

\section{RESULTS}

\section{Cloning GmDREB2 gene from Vietnamese local} soybean cultivars

GmDREB2 gene were amplified by RT- PCR from the soybean DT2008 and five different local soybean, which was $0.48 \mathrm{~kb}$ long, similar to the size of GmDREB2 gene published in GeneBank (DQ054363). GmDREB2 isolated from the local soybean cultivars shared 95 to $99.9 \%$ in sequence identity to DQ054363. The GmDREB2 sequences from these five local cultivars have been submitted on the GenBank with the codes LK936507, LK936508, LK936509, HG965097, HG965098, HG965099

(http://www.ncbi.nlm.nih.gov/nuccore). Among them, GmDREB2 from the cultivar DT2008 had the most different positions and had lower coefficient of similarity than DQ054363 and the others. Phylogenetic tree in Figure 1, based on the analysis of amino acid sequences, showed that nine soybean cultivars were divided into two main groups, the first group consisted of eight cultivars (CB, CBD, BG, BS, DT26, DT51, DVN5 and DQ054363) and the second one consisted of only DT2008; genetic distance between DT2008 and other cultivars in the group was $5.4 \%$. Thus, GmDREB2 was chosen from DT2008 to generate the recombinant protein for drought-tolerant purpose.

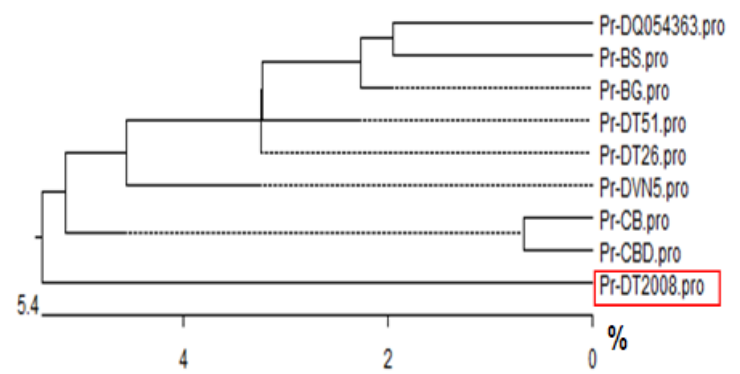

Figure 1 - Phylogenetic tree of soybean cultivars based on analysis of DREB2 amino acid sequence.

Construction of transgenic vector and the expression of the GmDREB2 gene

Designing structure 35S- GmDREB2-c-myc

GmDREB2 gene was isolated from the DT2008 drought-resistant soybean variety, and then was cloned into $\mathrm{pBT}$ vector. $p B T-G m D R E B 2$ and pRTRA7/3 vectors were cut by the restriction enzymes $N c o$ I and NotI (Fig. 2). These fragments were purified, and then ligated by ligase $\mathrm{T} 4$ to obtain pRTRA/35S-GmDREB2-c-myc. This result was checked by the PCR using specific primer pairs GmDREB2-NCoI/NotI.

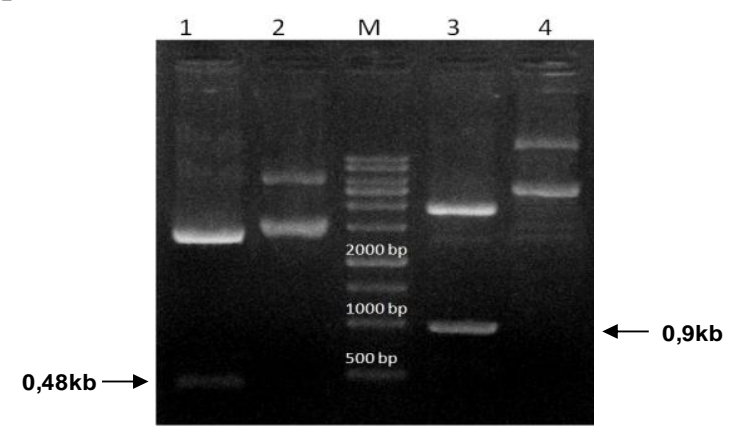

Figure 2 - Digested products of pBT-GmDREB2 and pRTRA $7 / 3$ by $N c o$ I and NotI. (M: DNA ladder $1 \mathrm{~kb}$; 1 - The cut pBT- GmDREB2 consisted of 2 fragments with the sizes of 500 bp and $2.7 \mathrm{~kb}$; 2- Size of pBT-GmDREB2 is 3 $\mathrm{kb}$; 3- The cut pRTRA consisted of 2 fragments with the sizes of $3.3 \mathrm{~kb}$ and $0.9 \mathrm{~kb}$; 4: Plasmid pRTRA7/3. 
Construction of transgenic vector carrying GmDREB2 gene

Structure 35S-GmDREB2-c-myc was ligated to pBI121 for the expression in the plants. pRTRA/35S-GmDREB2-c-myc and pBI121 was cut out using HindIII, Digestion of pRTRA/35SGmDREB2-c-myc produced two fragments with the sizes of $1.4 \mathrm{~kb}$ and $2.4 \mathrm{~kb}$, corresponding to 35S-GmDREB2- c-myc and pRTRA, respectively (Fig. 3A). 35S-GmDREB2-c-myc was cloned into
pBI121 vector, then transformed into $A$. tumefaciens by electroporation. The recombinant A. tumefaciens was checked by the PCR using specific primer pairs. The result showed that 500 $\mathrm{bp}$ fragments corresponding to DNA band of positive control sample were found in three bacterial strains (Fig. 3B), demonstrating that 35SGmDREB2-c-myc was transferred successfully into pBI121 vector. Figure $3 \mathrm{C}$ depicts the structure of this recombinant construct in $\mathrm{pBI} 121$ vector.

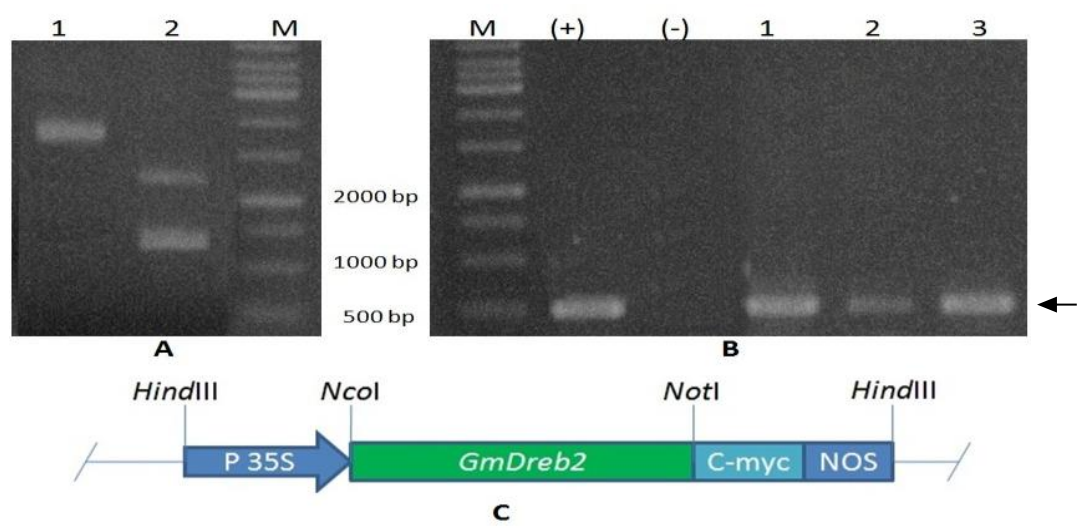

Figure 3 - A) pRTRA/35S-GmDREB2-c-myc vector (1); and digested products by HindIII (2). B) PCR products of GmDREB2 from A. tumefaciens CV58 habouring pBI121/35SGmDREB2-C-myc; C) 35S-GmDREB2-c-myc structure in pBI121.

The expression of the recombinant $\mathrm{DREB} 2$ protein in tobacco

35S-GmDREB2-c-myc was transformed into tobacco K326 using A. tumefaciens. PCR for the GmDREB2 gene from DNA extracted in the leaves revealed that 20 of 35 transgenic tobacco lines carried this transgene. Figure 4 illustrated the test result of 12 transgenic tobacco lines among 35 lines, in which the transgene was found in seven lines: TG2, TG3, TG5, TG6, TG8, TG9 and TG12.

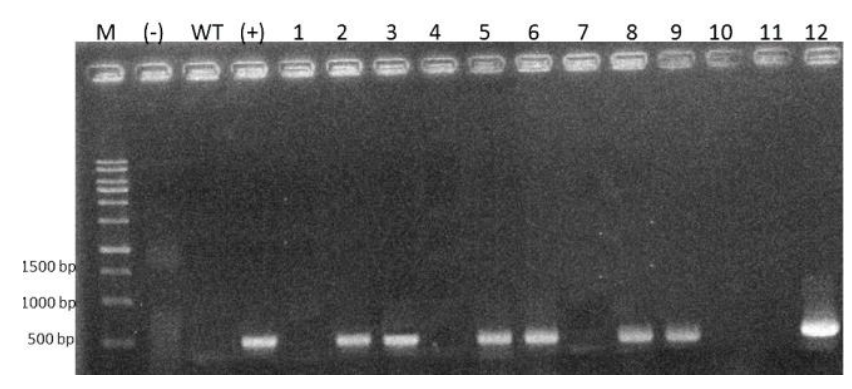

Figure 4 - PCR products of GmDREB2 transgene in 12 transgenic tobacco lines (M: DNA ladder $1 \mathrm{~kb}$; (-): negative control; (+): positive control; WT: wild type; 1- 12: tobacco transgenic lines).
Among them, five lines (TG2, TG3, TG6, TG8, and TG12) expressed the recombinant DREB2 protein as revealed by the Western Blot (Fig. 5).

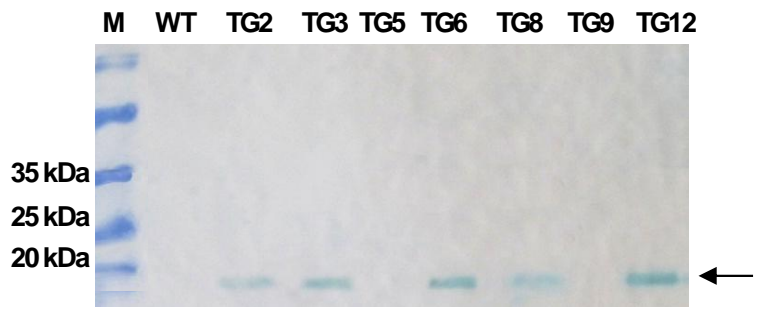

Figure 5 - GmDREB2 expression in wild type and transgenic tobacco plants $(\mathrm{M}$ : protein prestained marker $20-120 \mathrm{kDa}$; WT: Wild type; TG2, TG3, TG5, TG6, TG8, TG9, TG12: transgenic tobacco lines).

The proline concentration levels in the leaves of these five transgenic tobacco lines as well as in WT in normal water conditions and after five and nine days of drought stress are shown in Table 1. In normal water conditions, the transgenic lines had higher proline content than WT from $203.81 \%$ 
(TG3 line) to $245.72 \%$ (TG6 line). After artificial drought stress, both WT and transgenic tobacco lines had increased proline content; however, proline concentrations in the transgenic lines were higher as well. The rates of increase in the transgenic lines were higher than WT from 211.17 to $332.44 \%$ after five days of drought stress, and from 262.79 to $466.04 \%$ after nine days of drought stress. The two lines TG2 and TG12 had the highest rates of increase. After five days of drought stress, proline in WT increased $161.25 \%$, while in line TG2 and TG12, it increased 332.44 and $246.89 \%$, respectively. After nine days, proline in WT, TG2 and TG12 increased 212.27, 466.04 and $339.95 \%$, respectively. These results showed that the transgenic tobacco lines accumulated significantly more proline than WT under drought stress conditions. Thus, it could be concluded that the overexpression of GmDREB2 increased the amount of proline in response to drought-stress.

Table 1 - Proline concentration accumulated in transgenic tobacco lines and WT after five and nine days of drought stress

\begin{tabular}{|c|c|c|c|c|c|}
\hline \multirow{2}{*}{$\begin{array}{l}\text { Transgenic } \\
\text { tobacco lines } \\
\text { and WT }\end{array}$} & \multirow{2}{*}{$\begin{array}{l}\text { Normal conditions } \\
\qquad \begin{array}{c}(\mu \mathrm{mol} / \mathrm{gr}) \\
\bar{X} \pm \mathrm{S}_{\bar{X}}\end{array}\end{array}$} & \multicolumn{2}{|c|}{$\begin{array}{l}\text { The change in proline concentration } \\
5 \text { days after being drought stress }\end{array}$} & \multicolumn{2}{|c|}{$\begin{array}{c}\text { The change in proline concentration } 9 \\
\text { days after being drought stress }\end{array}$} \\
\hline & & $\begin{array}{l}\text { Concentration }(\boldsymbol{\mu m o l} / \mathrm{gr}) \\
\qquad \bar{X} \pm \mathrm{S}_{\bar{X}}\end{array}$ & $\begin{array}{c}\text { Rate of } \\
\text { increase }(\%)\end{array}$ & $\begin{array}{l}\text { Concentration }(\boldsymbol{\mu m o l} / \mathrm{gr}) \\
\qquad \bar{X} \pm \mathrm{S}_{\bar{X}}\end{array}$ & $\begin{array}{c}\text { Rate of } \\
\text { increase }(\%)\end{array}$ \\
\hline WT & $0.1076 \pm 0.0$ & $0.1735 \pm 0.0039$ & & $0.2284 \pm 0.0045$ & \\
\hline TG2 & $0.2232 \pm 0.0022$ & $0.7420 \pm 0.0052$ & 33 & $1.0402 \pm 0.0466$ & \\
\hline TG3 & $0.2193 \pm 0.0014$ & $0.4630 \pm 0.0055$ & 21 & $0.6583 \pm 0.0030$ & \\
\hline TG6 & $0.2644 \pm 0.0027$ & $0.6636 \pm 0.0038$ & 250.98 & $0.7567 \pm 0.0035$ & 286.20 \\
\hline TG8 & $0.2427 \pm 0.0020$ & $0.5184 \pm 0.0044$ & 213.60 & $0.6378 \pm 0.0029$ & 262.79 \\
\hline TG12 & $0.2508 \pm 0.0035$ & $0.6192 \pm 0.0040$ & 246.89 & $0.8526 \pm 0.0058$ & 339.95 \\
\hline
\end{tabular}

\section{DISCUSSION}

Abiotic stress is the main cause leading to crop damage in the world, reducing the yields of main crops more than 50\% (Boyer 1982; Bray et al. 1997). Among abiotic stresses, drought is a major factor limiting the crop yield. Analysis of morphoanatomical and micromorphometrical characteristics in soybean during water stress, Stolf et al. (2009) showed that differences in drought tolerance observed between the soybean cultivars seemed to be related to factors other than morphological traits since this species has a short lifecycle. According to the approach on the relation between genes and drought tolerance of plants, studies have provided advanced understanding of gene expression and signaling responses to drought in the plants (Zhu et al. 2010). Many drought-related responses of multiple genes involve proline synthesis (Chu et al. 2010), root elongation genes (Lo et al. 2014), and many other functional genes. A variety of plant genes induced by the drought and cold stress are thought to be involved in the stress tolerance. They are activated by the transcription factors, including DREB protein (Shinozaki et al. 1996). Overexpression of these transcription factors is a promising approach for the development of crop varieties resistant to drought and may be better than overexpression of a single functional gene (Bartels and Hussain 2008). DREB proteins belong to a protein family of transcription factors AP2/ERF, which regulate the expression of many genes induced by drought, salinity and low temperature in many plant species. DREB2 is one of 10 members of the DREB subfamily genes identified in the genome of soybean (Riechmann et al. 2000; Mizoi et al. 2012). DREB2 gene encoding a protein related to DRE/CRT, which is stimulated by osmotic stress (Liu et al. 1998). In this study, six GmDREB2 gene sequences were isolated from the Vietnamese local soybean cultivars, with the size of 480 nucleotides, encoding for 159 amino acids. The results of the AP2 region of DREB2 in the Vietnamese soybean cultivars and the previously published DQ054363 showed that AP2 consisted of 59 amino acids, from position 34 to position 92 with high similarity. Results from sequence comparison of GmDREB2 gene in six local soybean cultivars DT2008, CB, CBD, DT26, DT51, DVN5 and the previous published DQ054363 (GenBank) revealed that there were 26 different positions in the nucleotide sequence and 17 positions in the 
amino acid sequence. The cultivar DT2008 had the most difference compared to DQ054363 and other local cultivars. There was no difference in the amino acid sequence in the AP2 domain of these six local cultivars. However, this study did not identify any correlation between the difference in nucleotide and amino acid sequences of DREB2 and the drought tolerance of soybean plants. This could be interesting idea and should be studied further to clarify this correlation. It was found that AP2 domain was highly conserved, and the modification in this domain could alter protein properties. According to previous reports, DT2008 has the highest drought tolerance among these local cultivars (Mai et al. 2010; Ha et al. 2012; Sulieman et al. 2015). Thus, the GmDREB2 gene isolated from the DT2008 was used to design recombinant structures and generate transgenic tobacco plants, which resulted five transgenic lines expressing the recombinant DREB2 protein. These transgenic tobacco lines had higher proline accumulation than WT plants from 211.17 to $332.44 \%$ after five days of drought stress, and from 262.79 to $466.04 \%$ after nine days of drought stress. In higher plants, proline is synthesized from glutamate or ornithine. The synthesis of proline from glutamate is the most important cycle with the participation of the pyrroline-5-carboxylate synthetase (P5CS) and pyrroline-5-carboxylate reductase (P5CR) (Delauney and Verma 1993). The increase of the proline content in the transgenic tobacco compared to WT under drought stress conditions is possibly due to the overexpression of GmDREB2 transgene, which enhances the transcription of P5CS and P5CR gene, leading to the increase in proline content in transgenic plants and in turn improves the drought tolerance. The present results from the overexpression of GmDREB2 in the transgenic tobacco plants provided the basis for generating drought-tolerant transgenic soybean cultivars by GmDREB2 overexpression.

\section{CONCLUSIONS}

GmDREB2 gene from the soybean cultivar DT2008 and five Vietnamese local soybean cultivars (DT26, DT51, DVN5, CB, and CBD) was amplified and cloned. The coding region of GmDREB2 gene was isolated from six soybean cultivars, which contained 480 nucleotides in length, encoding 159 amino acids. AP2 domain of
DREB2 protein was 59 amino acids in length and contained DNA binding sites, including 11 amino acids. The transgenic vector containing the recombinant 35S- GmDREB2- c-myc structure was designed and transformed into tobacco plants. Recombinant DREB2 protein was expressed in five transgenic tobacco lines with a molecular weight of approximately $20 \mathrm{kDa}$. Transgenic lines had significantly higher proline concentrations than WT. Thus, GmDREB2 could be expressed in soybean to improve the drought tolerance of the soybean cultivars in Vietnam.

\section{REFERENCES}

Andeani JK, Mohsenzadeh S, Mohabatkar H. Isolation and characterization of partial DREB gene from four Iranian Triticum aestivum cultivars. World J Agric Sci.2009;5(5):561-566.

Bates LS, Waldren RP, Teare ID. Rapid determination of free proline for water-stress studies. Plant Soil. 1973;39 (1): 205-207

Bartels D, Hussain SS. Current status and implications of engineering drought tolerance in plants using transgenic approaches. $C A B$ int. 2008; 3 (020); DOI: 10.1079/PAVSNNR20083020 2008.

Boyer JS. Plant Productivity and Environment. Science. 1982;218 (4571): 443-448.

Bray EA. Plants responses to water deficit. Trends Plant Sci. 1997;2 (2): 48-54.

Chen M, Wang QY, Cheng XG, Xu ZS, Li LC, Ye XG, et al. GmDREB2, a soybean DRE-binding transcription factor, conferred drought and high-salt tolerance in transgenic plants. Biochem Biophys Res Commun. 2007; 353(2): 299-305.

Chu MH, Nguyen THT, Chu LH, Nguyen AT, Le SV, Chu HH. Characteristics of the gene encoding pyrroline-5-carboxylate synthase (P5CS) in Vietnamese soybean cultivars (Glycine max L. Merrill). 2010 International Conference on Biology, Environment and Chemistry. 2010; 319-323.

Delauney AJ, Verma DPS. Proline biosynthesis and osmoregulation in plants. The Plant J. 1993; 4 (2): 215-223.

Fujita Y, Fujita M, Satoh R, Maruyama K, Mohammad MP, et al. AREB1 Is a Transcription Activator of Novel ABRE-Dependent ABA Signaling That Enhances Drought Stress Tolerance in Arabidopsis. Plant Cell. 2005;17 (12): 3470-3488.

Ha CV, Le DT, Nishiyama R, Watanabe Y, Tran UT, Dong NV, et al. Characterization of the newly developed soybean cultivar DT2008 in relation to the model variety W82 reveals a new genetic resource for comparative and functional genomics for improved drought tolerance. Biomed Res Int. 2013; 759657. 
Kobayashi F, Ishibashi M, Takumi S. Transcriptional activation of Cor/Lea genes and increase in abiotic stress tolerance through expression of a wheat DREB2 homolog in transgenic tobacco. Transgenic Res. 2008; 17 (5): 755-767.

Li XP, Tian AG, Luo GZ, Gong ZZ, Zhang JS, Chen SY. Soybean DRE-binding transcription factors that are responsive to abiotic stresses. Theor Appl Genet. 2005;110(8):1355-1362.

Liu Q, Kasuga M, Sakuma Y, Abe H, Miura S, Yamaguchi-Shinozaki K, et al. Two transcription factors, DREB1 and DREB2, with an EREBP/AP2 DNA binding domain separate two cellular signal transduction pathways in drought- and lowtemperature-responsive gene expression, respectively, in Arabidopsis. Plant Cell. 1998; 10:1391-1406.

Liu W, Feng F. Nicotiana tabacum DREB2 mRNA, complete cds, EU727156. GenBank 2008.

Lo ST, Le SV, Nguyen VTT, Chu MH. Cloning and designing vector carrying GmEXPl gene isolated from local soybean cultivar Sonla, Vietnam. Internat J Biosc Biochem Bioinformatics. 2014; 4(3): 191-194.

Mai VQ, Pham CTB, Nguyen MV, Le HAT. Results of research, creation, drought-tolerant soybean variety, DT2008. J Viet Agricultural Sci Technol. 2010; 6: 4650.

Mizoi J, Shinozaki K, Yamaguchi-Shinozaki K. AP2/ERF family transcription factors in plant abiotic stress responses. Biochim Biophys Acta. 2012; 1819 (2): 86-96.

Mizoi J, Ohori T, Moriwaki T, Kidokoro S, Todaka D, Maruyama $\mathrm{K}$, et al. $\operatorname{GmDREB} 2 A ; 2$, a canonical dehydration-responsive element binding protein2type transcription factor in soybean, is posttranslationally regulated and mediates dehydration-responsive element-dependent gene expression. Plant Physiol. 2013; 161(1): 346-361.

Riechmann JL, Heard J, Martin G, Reuber L, Jiang C, Keddie $\mathrm{J}$, et al. Arabidopsis transcription factors: genome-wide comparative analysis among eukaryotes. Science. 2000; 290 (5499): 2105-2110.

Shinozaki K, Yamaguchi-Shinozaki K. Molecular responses to drought and cold stress. Cur Opinion Biotechnol. 1996; 7(2): 161-167.
Sambrook J, Russell DW. Molecular Cloning: A laboratory Manual, $1^{\text {st }} \mathrm{ed}$. Cold Spring Harbor laboratory Press, Cold Spring Harbor, NY 2001.

Sakuma Y, Maruyama K, Osakabe Y, Qin F, Seki M, Shinozaki K, et al. Functional analysis of an Arabidopsis transcription factor, DREB2A, involved in drought-responsive gene expression. Plant Cell. 2006a;18(5):1292-1309.

Sakuma Y, Maruyama K, Qin F, Osakabe Y, Shinozaki $\mathrm{K}$, Yamaguchi-Shinozaki K. Dual function of an Arabidopsis transcription factor DREB2A in waterstress- responsive and heat-stress-responsive gene expression. Proc Natl Acad Sci. USA 2006b;103(49):18822-18827.

Stolf R, Medri ME, Pimenta JA, Torres MR, Dias J, Lemos NG, et al. Morpho-anatomical and micromorphometrical evaluations in soybean genotypes during water stress. Braz Arch Biol Technol. 2009; 52 (6): 1313-1331.

Sulieman S, Ha CV, Nasr Esfahani M, Watanabe Y, Nishiyama R, Pham BCT, et al. DT2008: A Promising New Genetic Resource for Improved Drought Tolerance in Soybean When Solely Dependent on Symbiotic N2 Fixation. Biomed Res Int. 2015; 687213

Topping JF. Tobacco Transformation. Plant Virology Protocols. Met Molecular Biol. 1998; 81: 365-372.

Wang N, Liu M, Cheng X, Ma Y. Glycine max DREB protein (DREB2) gene, complete cds. DQ054363. GenBank. 2006.

Yamaguchi-Shinozaki K, Shinozaki K. A novel cisacting element in an Arabidopsis gene is involved in responsiveness to drought, low-temperature or highsalt stress. Plant Cell. 1994; 6(2): 251-264.

Zhu J, Lee BH, Dellinger M, Cui X, Zhang C, Wu S, et al. A cellulose synthase-like protein is required for osmotic stress tolerance in Arabidopsis. Plant $J$. 2010; 63 (1): 128-140. 